\title{
Optimising Traffic Lights with Metaheuristics: Reduction of Car Emissions and Consumption
}

\author{
Jose Garcia-Nieto \\ Departamento de Lenguajes y \\ Ciencias de la Computacion \\ Malaga, Spain \\ Email: jnieto@lcc.uma.es
}

\author{
Javier Ferrer \\ Departamento de Lenguajes y \\ Ciencias de la Computacion \\ Malaga, Spain \\ Email: ferrer@1cc.uma.es
}

\author{
Enrique Alba \\ Departamento de Lenguajes y \\ Ciencias de la Computacion \\ Malaga, Spain \\ Email: eat@lcc.uma.es
}

\begin{abstract}
In last years, enhancing the vehicular traffic flow becomes a mandatory task to minimize the impact of polluting emissions and unsustainable fuel consumption in our cities. Smart Mobility optimisation emerges then, with the goal of improving the traffic management in the city. With this aim, we propose in this paper an optimisation strategy based on swarm intelligence to find efficient cycle programs for traffic lights deployed in large urban areas. In concrete, in this work we focus on the improvement of the traffic flow with the global purpose of reducing contaminant emissions $\left(\mathrm{CO}_{2}\right.$ and $\left.\mathrm{NO}_{x}\right)$ and fuel consumption in the analyzed areas. For the sake of standardization, we follow European Union reference framework for traffic emissions, called HandBook Emission FActors (HBEFA). As a case study, we have concentrated in two extensive urban areas in the cities of Malaga and Seville (in Spain). After several comparisons between different optimisation techniques (Differential Evolution and Random Search), as well as other solutions provided by experts, our proposal is shown to obtain significant reductions of fuel consumption and gas emissions.
\end{abstract}

\section{INTRODUCTION}

In last decades, the high level of pollution in the air and the hydrocarbons consumption derived from the urban traffic are becoming serious issues, since they affect the citizens' health, the global economy, and the difficulty of city management. Hence, the improvement of vehicle mobility is a key task in urban areas, and might have a positive impact in the efficacy of traffic flow management. However, this is not always affordable due to the cost of new physical infrastructures (e.g., info LED panels). Therefore we address our efforts towards an efficient generation of cycle programs of traffic lights. In this sense, we provide a low cost alternative to alleviate city traffic flow [13], [14], [15]. Nevertheless, the number of traffic lights is rising in modern cities, and its scheduling is becoming more and more complex due to their huge number of combinations that should be considered. Thus, the use of automatic intelligent tools for the optimal cycle programming of traffic lights is an essential task in traffic flow management.

In this sense, the application of intelligent optimisation techniques, like metaheuristic algorithms [1], has become important since their adequacy has been already demonstrated for the generation of traffic lights programs [6], [15], [17] with the aim of enhancing the global traffic flow. However, to the best of our knowledge, there is no related work focusing on the environmental perspective, with the aim of reducing contaminant emissions and fuel consumption: this is what we do in this paper. In addition, in most cases, the use of such intelligent systems is limited to the optimisation of small instances with only one or two junctions and 8 traffic lights at most, which is far from the study of whole cities.

The main motivation of this work is therefore to propose an optimisation strategy based on a Particle Swarm Optimisation algorithm (PSO) [10] named PSO-tl, to efficiently obtain cycle programs of traffic lights from an environmental perspective, with the target of reducing $\mathrm{CO}_{2}$ and $\mathrm{NO}_{x}$ emissions, as well as the total fuel consumption of vehicles. Moreover, we optimise the cycle programs of big urban areas with a large number of traffic lights and vehicles, showing that metaheuristics are strongly advisable techniques for future development in this domain.

PSO is an easily configurable algorithm, which in general, develops a quick convergence towards high quality solutions [10]. In fact, this is a desirable characteristic, since it allows us to obtain good solutions with fast techniques. For the evaluation of the cycle programs (coded as vector solutions), we have used the Simulator of Urban Mobility (SUMO) [11]. From the simulator we obtain a continuous source of information about the vehicle flow. This is a key aspect when using an advanced algorithm to generate automatic timing programs for traffic lights. In addition, SUMO allows us to work with the standard model of emissions HBEFA [9], by which we obtain the information about pollution and fuel consumption. This valuable information is used by the algorithm to evaluate candidate solutions.

In this study we have used two scenario instances located in the city downtown of Malaga and Seville (Spain), with hundreds of traffic lights and different traffic densities (250 and 500 vehicles). The experimental comparisons of our PSO-tl with other techniques such as Differential Evolution(DE) [18], Random Search (RANDOM), and Sumo Cycle Program Generator (SCPG) [11], will show with no doubt the significant improvement obtained by our proposal in terms of $\mathrm{CO}_{2}, \mathrm{NO}_{x}$ emissions (which are the most commonly found compounds in tailpipe's concentration tests of vehicles with gasoline and diesel engines), and fuel consumption.

The remainder of the article is organized as follows. Next section presents an overview of related works in the current literature. In Section III, the cycle program optimisation problem and the standard model of emissions HBEFA are described. After this, Section IV introduces our optimisation strategy, paying special attention to the fitness function based on en- 
vironmental factors. Section $\mathrm{V}$ is devoted to the experiments and the analysis of the solutions. Finally, Section VI outlines some conclusions and future work.

\section{RELATED WORK}

Several works can be found in the related literature that deal with the traffic congestion problem by means of accurate signal lights timing programs [12], [16]. We are mostly focusing on those studies that use Metaheuristic approaches [2] for optimising traffic light staging problems.

A first attempt was proposed by Rouphail et al. [19], where a Genetic Algorithm (GA) was coupled with the CORSIM [7] microsimulator for the timing optimisation of nine intersections in the city of Chicago (USA). Following the model proposed in Brockfeld et al. [3], Sánchez et al. [15] designed a GA with the objective of optimising the cycle programming of traffic lights in a commercial area in the city of Santa Cruz de Tenerife (Spain). In that approach, the computation of valid states was done before the algorithm began, and it highly depended on the scenario instance tackled. A GA was also used by Turky et al. [21] to improve the performance of traffic lights and pedestrian crossing control in a single four way, two lane intersection.

Peng et al. [17] presented a Particle Swarm Optimisation (PSO) with isolation niches for the scheduling of traffic lights. In that approach, a purely academic instance with a restrictive one-way road with two intersections was used to test the proposal. Kachroudi and Bhouri [8] applied a multiobjective version of PSO for optimising cycle programs using a predictive model control based on a public transport progression model. In that approach, private and public vehicle models were used to carry out simulations on a virtual urban road network made up of 16 intersections and 51 links.

These studies have focused on different aspects of the traffic light programming. However, a common limitation to all of them is that they considered just a small scenario with specific traffic conditions. This means that the resulting cycle programs might not scale to larger instances. More recently, García-Nieto et al. [6], [5] proposed a PSO approach with SUMO microsimulator for traffic light programming in large realistic urban areas. In this work, the global journey time and the number of vehicles that reach their destinations in observation time.

In general, all the approaches mentioned above are mainly focused on global trip times and waiting times of vehicles in traffic lights, although none of them considered the influence of pollutant emissions and fuel consumption factors in solutions.

In contrast, our proposal deals with the optimisation of several emission factors: $\mathrm{CO}_{2}, \mathrm{NO}_{x}$, and fuel consumption, for large realistic urban areas, with the aim of providing the experts with optimised traffic lights programs oriented to environmental savings.

\section{How to Define Cycle PROgRams}

In a road network, the traffic lights are located in the intersections. The traffic flow is controlled according to the cycle programs and their phase duration. In an intersection, all traffic lights are controlled by a common cycle program because they should be synchronized for security reasons. In addition, for all traffic lights in an intersection, the color combination during their phase must be always valid, obeying real traffic rules with the aim of avoiding collisions and accidents, in general. In our model, we just consider valid combinations of color states for any intersection, which are constant during all the optimisation process. In this way, we avoid invalid combinations of color states, while at the same time, it also restricts the search to only valid states.

In this context, our main objective consists in finding optimised cycle programs for all traffic lights located in a specific urban area. A cycle is defined as the period of time, in which a set of traffic lights in the same intersection has the same color state. In addition, these cycle programs should be coordinated with the traffic lights of the adjacent intersections, with the intention of improving the traffic flow according to the provisions of road traffic regulation.

\section{A. Encoding}

Following the SUMO's specification for programming cycles, we have encoded solutions as vectors of integers. In this way, each element of the vector (variable) represents the phase duration (color combination) of the traffic lights involved in a particular intersection. Fig. 1 shows an example of encoded solution that represents the intersection " $i$ ", which has 12 signals (colors) with current state " $G r r$ GGGr $r r G G G$ ", meaning that seven traffic lights are green $7(G)$, and the other six ones are red $(r)$ for 36 seconds. In this way, the next phase changes the traffic lights state to another valid combination “ $y G G$ rrry GGr $y y$ " (being ' $y$ ' amber), for 6 seconds, and so on. Once the last phase of an intersection is finished (the number of phases of each intersection could be different, it depends on the number of traffic lights and their location), the first phase will be the next one, i.e. the last phase is followed by the first one, and this cycle is repeated for the entire work of a city. Our representation allows us to take into account the interdependence between variables, not only for the phase duration in the same intersection, but also for phases of traffic lights in adjacent intersections.

In addition, SUMO allows us to simulate environmental factors based on the HBEFA model (HandBook Emission Factors for Road Transport) [9]. By using this model, we are able to simulate different vehicle characteristics such as accelerating, decelerating, braking, maximum speed, as well as their implications in the emissions based on the HBEFA model: hydrocarbons, particles, $\mathrm{CO}, \mathrm{CO}_{2}, \mathrm{NO}_{x}$ levels, and other pollution agents. In this study we have focused on the following contaminating agents: $\mathrm{CO}_{2}$ and $\mathrm{NO}_{x}$, as well as fuel consumption $(F u e l=F)$.

\section{B. Fitness Function}

In order to evaluate the generated cycle programs we have defined the fitness function $f(s)$ in Equation 1. It considers the traffic information obtained by the simulation with real data of the city.

$f(s)=\frac{\left\|\sum_{V_{l}} C O_{2}(s)\right\|_{l}+\left\|\sum_{V_{l}} N O_{x}(s)\right\|_{l}+\left\|\sum_{V_{l}} F(s)\right\|_{l}}{\# \text { edges }}+\rho \cdot \bar{V}(s)$ 


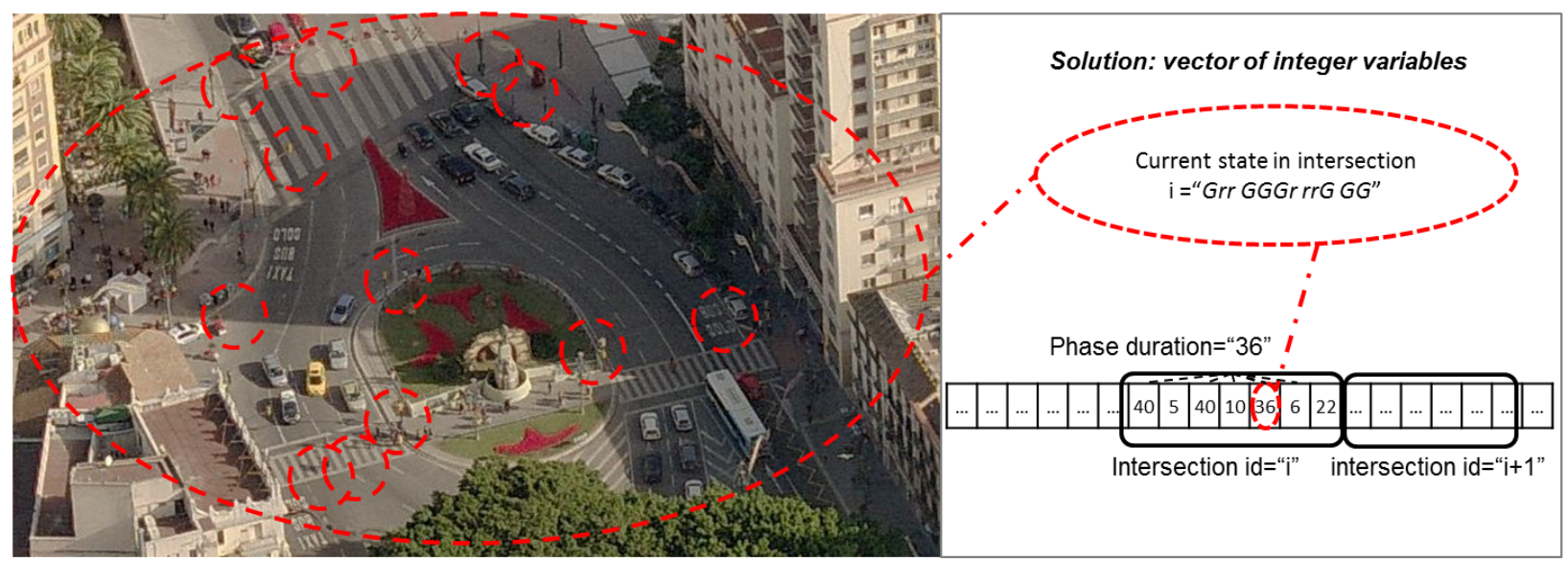

Fig. 1. Cycle program (phase duration) of traffic lights in a intersection

The main objective consists of minimizing the emission levels and the general fuel consumption of the studied instances. Equation 1 considers all the vehicles $(V)$ emissions indexes, normalized by the length of the street $(l)$ where the values were obtained, that is $\mathrm{CO}_{2}$ and $N O_{x}$ in $\mathrm{mg} / \mathrm{s}$, and the fuel consumption $F$ in 1/s. This sum of three terms is divided by only the number of streets (\#edges) which were transited by the vehicles. Finally, we have also considered the vehicles that do not arrive at their destination in the study time $(\bar{V})$, by multiplying them by an average of the environmental cost $\rho$. The use of the $\rho$ constant is necessary in order to compute the environmental indicators for the vehicles that are stuck in traffic (do not arrive at their destination in simulation time). We have set the environmental cost of the vehicles that do not arrive at their destination to be $\rho=0.75$ as an approximation of the average journey cost in different traffic conditions.

\section{OPTIMISATION STRATEGY}

Our optimisation strategy follows two stages: fist, we use our particular implementation of a Particle Swarm Optimisation for traffic lights (PSO-tl) to generate optimal cycle programs to reduce the vehicular contaminant emissions. Second, we gather data from the SUMO micro-simulator to enhance the optimisation process with the information extracted from the simulation of the realistic scenarios.

- PSO-tl. This is a population-based metaheuristic algorithm that follows the search procedure induced of the Standard PSO 2011 [4]. Besides, we have adapted the algorithm in order to find optimal (or quasi-optimal) cycle programs for traffic lights. In PSO-tl, the initial swarm is composed of a number of particles (solutions) initialized with a set of random values representing the phase durations. These values are within the time interval $[5,60] \in N$, and constitute the range of possible time spans (in seconds) a traffic light can be kept on a signal color (only green or red, the time for amber is a constant value). We have specified this interval by following several examples of real traffic light programs provided by the Malaga's City Council (Spain).

In PSO-tl, each potential solution to the problem is called a particle position and the population of particles is called the swarm. In this algorithm, each particle position $x^{i}$ is updated each iteration $g$ by means of the Equation 2.

$$
x_{g+1}^{i}=x_{g}^{i}+v_{g+1}^{i}
$$

where the term $v_{g+1}^{i}$ is the velocity of the particle, given by Equation 3.

$$
v_{g+1}^{i}=w \cdot v_{g}^{i}+G r_{g}^{i}-x_{g}^{i}+H S\left(G r,\left\|G r-\overrightarrow{x_{g}}\right\|\right)
$$

where $w$ is the inertia weight of the particle (it controls the trade-off between exploration and exploitation) and $G r_{g}^{i}$ is given by the following equation:

$$
G r_{g}^{i}=\frac{x_{g}^{i}+p_{g}^{\prime i}+l_{g}^{i}}{3},
$$

where $p_{g}^{i}$ is the best solution that the particle $i$ has seen so far, given by Equation 5, and $l_{g}^{\prime i}$ is the best particle of a neighborhood of $k=3$ (also known as the social best) randomly (uniformly) selected from the swarm, given by Equation 6

$$
\begin{aligned}
& p_{g}^{\prime i}=x_{g}^{i}+c \cdot\left(p_{g}^{i}-x_{g}^{i}\right) \\
& l_{g}^{\prime i}=x_{g}^{i}+c \cdot\left(l_{g}^{i}-x_{g}^{i}\right)
\end{aligned}
$$

where the acceleration coefficient $c>1$ is a uniform random value obtained from a distribution with $\mu=1 / 2$ and $\rho=1 / 12$. This coefficient is sampled a new for each component of the velocity vector.

Finally, $H S$ is a random number generator in a hypersphere, with $G r$ as gravity centre, i.e., $G r$ is calculated as the equidistant point between $p_{g}^{\prime}, l_{g}^{\prime}$ and $x_{g}$. This mechanism helps us to be independent from the coordinate system, improving this aspect in contrast to the previous versions of PSO [4].

Since optimal cycle programs require solutions encoded within a vector of positive integers (representing phase durations in seconds), we have used the quantisation method provided in the standard specification of PSO 2011 [4]. This quantisation is applied to each new generated particle, and transforms the continuous values of particles to discrete ones. 


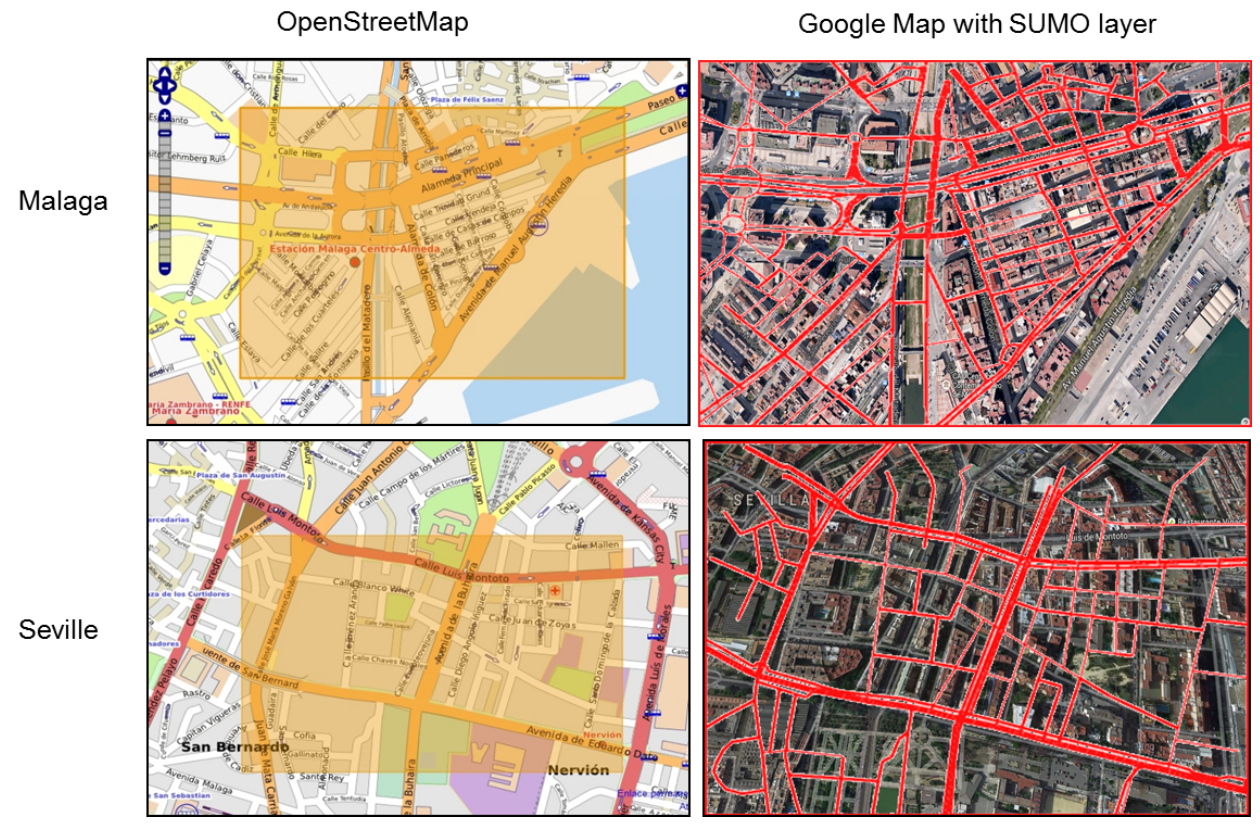

Fig. 2. Malaga and Seville instances exported from OpenStreetMaps and imported to SUMO

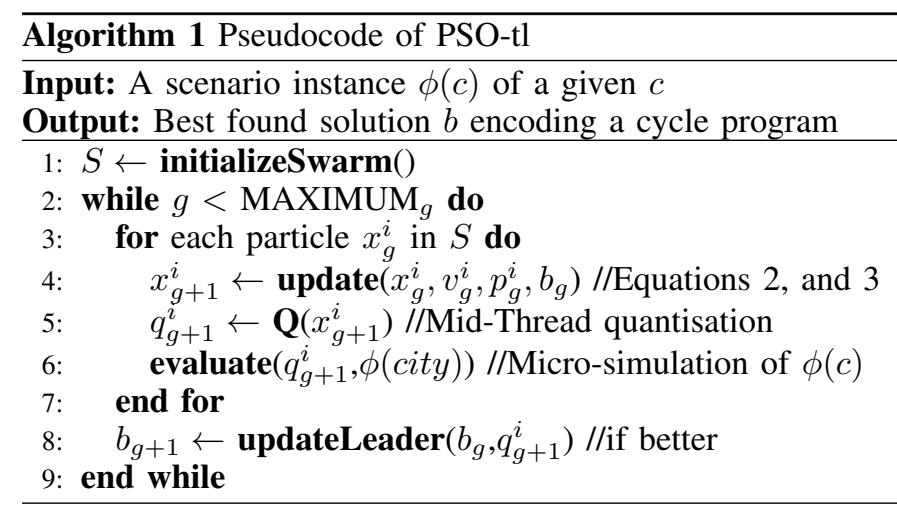

It consists of a Mid-Thread uniform quantiser as specified in Equation 7. The quantum step is set here to $\Delta=0.5$.

$$
Q(x)=\Delta \cdot\lfloor x / \Delta+0.5\rfloor
$$

The pseudo-code of PSO-tl is introduced in Algorithm 1. The algorithm starts by initializing the swarm (Line 1), which includes both the positions and velocities of the particles. The corresponding $p^{i}$ of each particle is randomly initialized, and the leader $b$ is computed as the best particle of the swarm. Then, for a maximum number of iterations, each particle is updated (Line 4), quantised (Line 5), and evaluated (Line 6), according to a scenario instance $\phi(c i t y)$. At the end of each iteration, the leader $b$ is also updated (Line 8). Finally, the best solution (cycle program in individual $b$ ) found so far is returned.

The simulation procedure is then used for assigning a quantitative quality value (fitness) to the solutions, thus leading to optimised cycle programs tailored to a given urban scenario instance. This task is tackled by the SUMO microscopic traffic simulator. When PSO-tl generates a new solution, it is used for updating the cycle program (candidate solution). Then, SUMO is started to simulate the instance with streets, directions, obstacles, traffic lights, vehicles, speed, routes, etc., under different traffic patterns, and with the new defined schedule of cycle programs. After the simulation, SUMO returns the global information necessary to compute the fitness function (Equation 1), particularly the data from emissions and fuel consumption for each vehicle in each street. The new cycle program is statically loaded for each simulation procedure, and what real traffic light human schedulers actually demand are constant cycle programs for specific areas and for preestablished time periods (rush hours, night periods, etc.), which led us to take this focus.

\section{EXPERIMENTS}

In this work, we have used two traffic scenarios with information obtained from real digital maps (illustrated in Fig. 2). These scenarios are located in urban areas of approximately $750 \mathrm{~m}^{2}$ of Malaga and Seville cities. The information used concerns: traffic rules, traffic element locations, buildings, road directions, streets, intersections, etc. Moreover, we have set the number of vehicles circulating, as well as their speeds, by following current specifications available in the Mobility Delegation of these cities ${ }^{1}$. These areas have the following characteristics:

1) Malaga. In the zone between Andalucia and Aurora avenues, this scenario (Fig. 2, top) is composed by streets with different widths and lengths, and several roundabouts. It contains junctions including from 4 to 16 traffic lights each one.

2) Seville. Located in the popular district of Nervion in the city center of Seville (Fig. 2, bottom), it is

\footnotetext{
${ }^{1}$ Malaga (http://movilidad.malaga.eu/), Seville (http://www.trajano.com/). This information was collected from sensorized points in certain streets obtaining a measure of traffic density at several time intervals.
} 
made up of intersections between streets including each one from 4 to 17 traffic lights. The complete area shows a representative organization with almost all the junctions connecting between three and four streets.

The number of studied intersections is 70 for the two instances. We have to notice that in spite of having in both instances a similar number of intersections (70), the number of signal lights is not exactly the same, since they contain different intersection shapes (312 traffic lights in Malaga and 358 ones in Seville). In addition, for each scenario we have generated two instances with different traffic densities, 250 and 500 vehicles circulating, in a simulation time of 1,200 seconds (micro-simulation iterations). This time was determined as a maximum time for a car to complete its route, even if it must stop in all the traffic lights it finds. Each vehicle performs its own route from its own origin to destination circulating with a maximum speed of $50 \mathrm{~km} / \mathrm{h}$ (typical in urban areas). The routes were previously generated by following random paths.

\section{A. Experimental Setup}

We have implemented our PSO-tl in $\mathrm{C}++$ following the skeleton structure of the MALLBA framework of metaheuristics [1]. The simulation phase is carried out by executing (for the evaluation of particles) the traffic simulator SUMO release 0.12.0 for Linux. All the executions were run in a cluster of 16 machines with Intel Core2 Quad processors Q9400 (4 cores per processor) at $2.66 \mathrm{GHz}$ and $4 \mathrm{~GB}$ memory running Ubuntu 12.04.1 LTS and managed by the HT Condor 7.8.4 cluster manager.

For each scenario instance we have carried out 30 independent runs of our PSO-tl. The swarm size is 30 particles, and we performed 300 optimisation iterations, resulting in a total of 9,000 evaluations in SUMO per execution and instance. As previously mentioned, the particle size directly depends on the number of phase timespans of traffic lights of each instance, being 304 and 368 for the instances of Malaga and Seville, respectively.

For the sake of a fair experimentation, we have also reimplemented two other algorithms also in the scope of the MALLBA library: the Differential Evolution (DE) [18] in its canonical version $(D E \backslash$ rand $\backslash 1)$ named Differential Evolution for traffic lights (DE-tl), and a Random Search Algorithm (RANDOM). Thus, by performing the same experimentation procedure, we expect to obtain some insights into the power of our proposal (how much intelligent it is) regarding a technique without any heuristic information in its operation (RANDOM), and with regards to another metaheuristic (DEtl). The maximum number of evaluations for these algorithms is also 9,000 .

Finally, we have added a fourth algorithm to the comparison. It is a deterministic algorithm provided by SUMO for generating cycle programs (SUMO Cycle Program Generator - SCPG). This last algorithm basically consists in assigning the phase durations to the intersections fresh values in the range of [6:31], according to three different factors the proportion of green states in the phases, the number of incoming lanes to the intersection, and the braking time of the vehicles approaching to the traffic lights. Further information about this algorithm can be found in [11]. Then, we also compare the cycle programs obtained by our PSO-tl against the ones obtained by SUMO.

\section{B. Experimental Results}

First of all, in this section we analyze the behaviour of our proposal from a computational performance perspective. In Table I we show the results of quality (fitness) obtained by our PSO-tl algorithm. These results are the Maximum, Median, Minimum, Mean, and Standard Deviation of the statistical distributions obtained for the Malaga and Seville scenarios (out of 30 independent run), with a traffic density of 250 and 500 vehicles. We also show the results obtained by the algorithms DE-tl, RAND, and SCPG. They have followed the same experimental procedure and been applied to the same scenario instances. In addition, in the same table, the last row for each instance shows the result of the statistical tests (nonparametric) [20] of Friedman and Holm to determine which algorithm is the best (ranking) and whether or not there exists significant differences between the best one (control) and the others. Specifically, these results are the p-values adjusted by the Holm test $\left(H A_{p}\right)$ for a statistical confidence level of $\alpha=0.05$.

\section{Performance Comparisons}

As it can be noticed, in Table I, the PSO-tl approach obtains the best fitness value (minimization) for all traffic scenarios. The difference is statistically significant between the resulting distributions. Although there exist two exceptions, one for the Seville scenario with 250 vehicles, in which, the results of DEtl are statistically similar ( $p$-value $=7,18 E-02)$ in comparison with PSO-tl. The other exception is for Malaga with 500 vehicles, where the results of DE-tl are statistically equivalent ( $p$-value $=1,09 E-01)$, but with a worse ranking for DE-tl in both cases. In particular, the DE-tl algorithm is the second best in ranking, followed by RAND and SCPG.

In this sense, another interesting observation consists in the fact that all search-based algorithms with a stochastic component obtain, in general, better results that the algorithm suggested by the experts (SCPG), which is deterministic. Even the RAND algorithm generates, after several simulations, better cycle programs for traffic lights than SCPG. This means that the problem is highly difficult and lack of clear patterns for experts when we go to a large scale optimisation scenario. These results give us some confidence in our optimisation strategy, since in most experiments, PSO-tl provided us quantitative benefits with respect to the method used by the experts (i.e., it is very robust).

\section{Environmental Factors Analysis}

In this section we analyze the direct impact of our proposed solutions over the studied environmental factors. To do so, we use the cycle programs obtained in the experiments.

As aforementioned, according to the HBEFA model [9], the two chemical compounds $\mathrm{CO}_{2}$ and $\mathrm{NO}_{x}$ are the most commonly found in tailpipe's concentration tests of vehicles with gasoline and diesel engines. Consequently, in this study we have computed the traces of the two chemical factors generated by all vehicles in the two scenarios. Fig. 3 (right) shows 
TABLE I. Quality VAlues obtained in Malaga AND SEVILle With 250 AND 500 VEHICLES

\begin{tabular}{|c|c|c|c|c|c|c|c|c|c|}
\hline \multirow{3}{*}{ City } & \multirow{3}{*}{ Value } & \multicolumn{8}{|c|}{ Traffic Density (Number of Vehicles) } \\
\hline & & \multicolumn{4}{|c|}{250} & \multicolumn{4}{|c|}{500} \\
\hline & & PSO-tl & DE-tl & RAND & SCPG & PSO-tl & DE-tl & RAND & SCPG \\
\hline \multirow{6}{*}{ Malaga } & Maximum & $1,04 \mathrm{E}+02$ & $1,08 \mathrm{E}+02$ & $1,11 \mathrm{E}+02$ & $1,05 \mathrm{E}+02$ & $2,95 \mathrm{E}+02$ & $3,06 \mathrm{E}+02$ & $3,42 \mathrm{E}+02$ & $5,59 \mathrm{E}+02$ \\
\hline & Median & $9,76 \mathrm{E}+01$ & $1,02 \mathrm{E}+02$ & $1,08 \mathrm{E}+02$ & $1,05 \mathrm{E}+02$ & $2,41 \mathrm{E}+02$ & $2,62 \mathrm{E}+02$ & $3,07 \mathrm{E}+02$ & $5,59 \mathrm{E}+02$ \\
\hline & Minimum & $9,12 \mathrm{E}+01$ & $9,83 \mathrm{E}+01$ & $1,04 \mathrm{E}+02$ & $1,05 \mathrm{E}+02$ & $1,92 \mathrm{E}+02$ & $2,25 \mathrm{E}+02$ & $2,66 \mathrm{E}+02$ & $5,59 \mathrm{E}+02$ \\
\hline & Mean & $9,72 E+01$ & $1,02 \mathrm{E}+02$ & $1,08 \mathrm{E}+02$ & $1,05 \mathrm{E}+02$ & $2,41 E+02$ & $2,64 \mathrm{E}+02$ & $3,04 \mathrm{E}+02$ & $5,59 \mathrm{E}+02$ \\
\hline & Standard Deviation & $3,00 E+00$ & $1,93 \mathrm{E}+00$ & $1,50 \mathrm{E}+00$ & $0,00 \mathrm{E}+00$ & $2,50 \mathrm{E}+01$ & $1,94 \mathrm{E}+01$ & $1,95 \mathrm{E}+01$ & $0,00 \mathrm{E}+00$ \\
\hline & $H A_{p}$ & - & $1,42 E-02$ & $1,78 E-08$ & $1,33 E-16$ & - & $1,09 E-01$ & $5,20 E-06$ & $5,49 E-16$ \\
\hline \multirow{6}{*}{ Seville } & Maximum & $2,04 E+02$ & $2,19 \mathrm{E}+02$ & $2,16 \mathrm{E}+02$ & $3,96 \mathrm{E}+02$ & $5,89 \mathrm{E}+02$ & $5,91 \mathrm{E}+02$ & $5,58 \mathrm{E}+02$ & $7,27 \mathrm{E}+02$ \\
\hline & Median & $1,73 E+02$ & $1,89 \mathrm{E}+02$ & $2,10 \mathrm{E}+02$ & $3,96 \mathrm{E}+02$ & $4,74 \mathrm{E}+02$ & $5,00 \mathrm{E}+02$ & $5,34 \mathrm{E}+02$ & $7,27 \mathrm{E}+02$ \\
\hline & Minimum & $1,54 \mathrm{E}+02$ & $1,79 \mathrm{E}+02$ & $1,97 \mathrm{E}+02$ & $3,96 \mathrm{E}+02$ & $4,21 \mathrm{E}+02$ & $4,79 \mathrm{E}+02$ & $5,09 \mathrm{E}+02$ & $7,27 \mathrm{E}+02$ \\
\hline & Mean & $1,78 \mathrm{E}+02$ & $1,92 \mathrm{E}+02$ & $2,08 \mathrm{E}+02$ & $3,96 \mathrm{E}+02$ & $4,75 \mathrm{E}+02$ & $5,10 \mathrm{E}+02$ & $5,36 \mathrm{E}+02$ & $7,27 \mathrm{E}+02$ \\
\hline & Standard Deviation & $1,49 E+01$ & $9,08 \mathrm{E}+00$ & $5,79 \mathrm{E}+00$ & $0,00 \mathrm{E}+00$ & $3,17 E+01$ & $2,96 \mathrm{E}+01$ & $9,55 \mathrm{E}+00$ & $0,00 \mathrm{E}+00$ \\
\hline & $H A_{p}$ & - & $7,18 E-02$ & $1,52 E-07$ & $6,82 E-17$ & - & $9,32 E-03$ & $9,58 E-07$ & $1,89 E-17$ \\
\hline
\end{tabular}
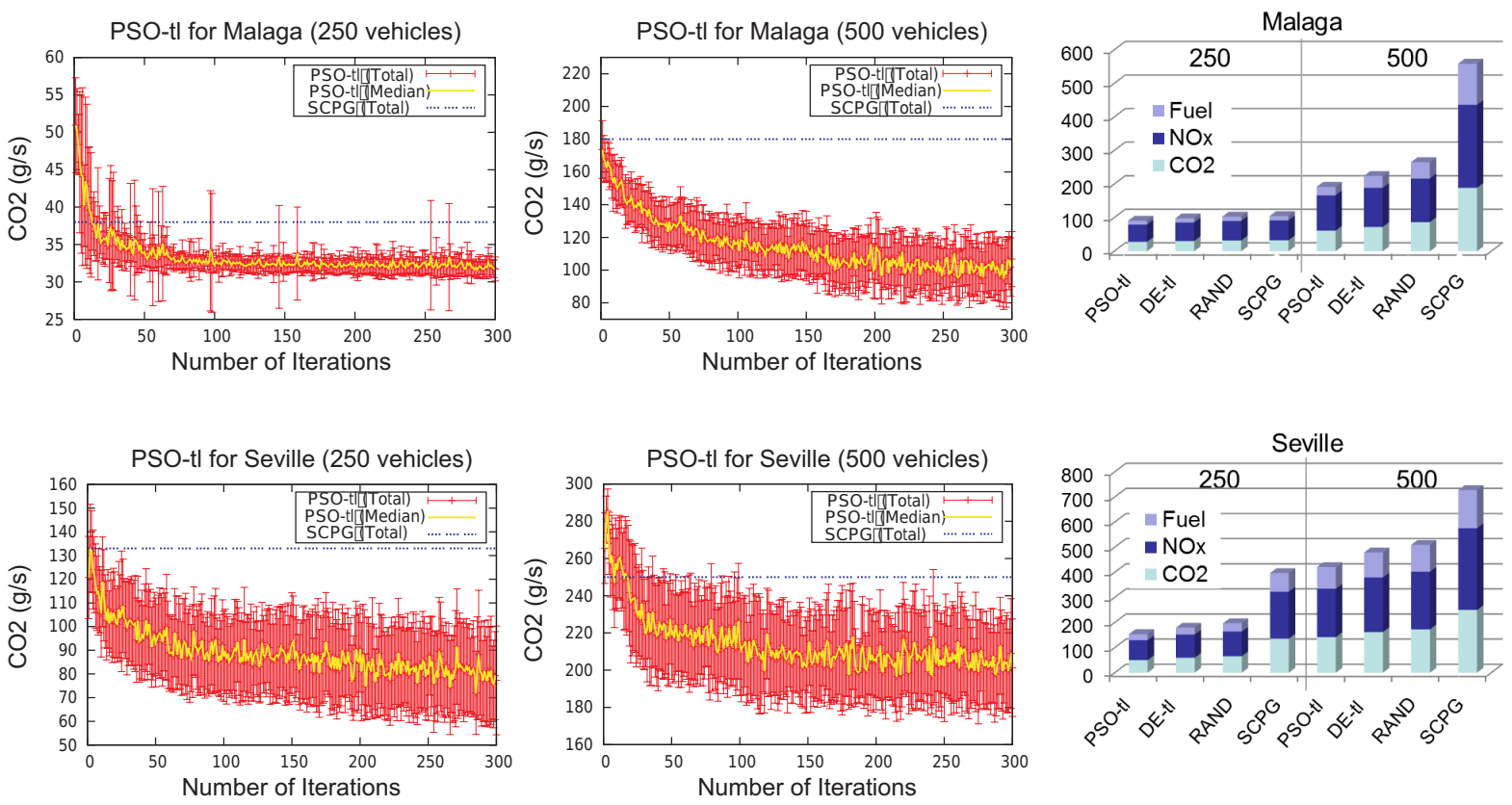

Fig. 3. Emissions of $\mathrm{CO}_{2}$ (first and second column from the left) and environmental cost for all algorithm (right)

the bars graph of the general concentrations of $\mathrm{CO}_{2}(\mathrm{~g} / \mathrm{s})$ and $N O_{x}(\mathrm{~g} / \mathrm{s})$ of the best cycle programs generated by each algorithm, for the Malaga and Seville scenarios. In addition, we also show the values of hydrocarbons consumption (Fuel en $1 / \mathrm{s})$.

In general, we observe that the environmental cost of the cycle programs generated by PSO-tl are lower than the ones generated when following other strategies.. While SCPG obtains low values of solution quality in the factors of pollution and consumption, the differences with regards to the other approaches are even higher when there are a high density of vehicles (500). This fact allows us to guess that PSO-tl might obtain even larger differences in high scale scenarios with thousands of vehicles. Especially, in the case of Seville with 500 vehicles, where the improvement obtained by PSOtl for $\mathrm{CO}_{2}$ emissions with regards to SCPG is 43.2\%; the improvement in terms of $N O_{x}$ is $40.9 \%$; and the improvement with respect to hydrocarbons consumption is $43.4 \%$. All of them mean highly important reductions of the experts' cycle programs.

In Fig. 3, we show the evolution graphs of $\mathrm{CO}_{2}$ emitted with cycle programs generated by PSO-tl with 250 (first column from the left) and 500 (second column from the left) vehicles, for Malaga and Seville. In the case of Malaga with 250 vehicles, we obtain a slight improvement in comparison with the solution proposed by the experts. However, when we simulate with twice this number of vehicles (500), we obtain a high improvement with regards to the SCPG solution. This behaviour is also observed in both instances of Seville. In addition, for this last city we observe a higher variability in the cycle programs obtained by PSO-tl than for the Malaga scenario. This fact gives us an idea of the difference of complexity of these two scenarios, showing seville as a more complex topology than Malaga. In any case, our proposal provides an important reduction of the level of pollution compared to the experts' solutions represented by a dotted line of SCPG results. 


\section{CONCLUSIONS}

In this paper, we propose an optimisation strategy based on PSO and a simulation with SUMO for the generation of cycle programs of traffic lights. Our main objective is to reduce the $\mathrm{CO}_{2}$ and $\mathrm{NO}_{x}$ emissions, as well as the consumption of hydrocarbons through the improvement of the traffic flow. As a case of study, we have used realistic models of urban areas of $0.75 \mathrm{~km}^{2}$ located in Malaga and Seville with traffic densities of 250 and 500 vehicles.

As main conclusions, the following ones could be extracted:

- Our optimisation proposal achieves significant improvements in terms of polluting emissions and consumption of hydrocarbons, in comparison with other search-based stochastic techniques (DE-tl and RAND), as well as with regards to expert's cycle programs (SCPG).

- The specific reduction of the three studied factors $\left(\mathrm{CO}_{2}, \mathrm{NO}_{x}\right.$, and Fuel $)$ depends on the instance, but they are always in the range $12 \%-43 \%$. Seville scenario seems to be more complex that Malaga due to its complex topology.

- If we extrapolate the results, this means that in a real case concerning the city of Malaga, with 600,000 inhabitants approximately, and 2.15 tons of $\mathrm{CO}_{2}$ per inhabitant/year, ${ }^{2}$ we will save around 510,000 tons of $\mathrm{CO}_{2}(40 \%$ less $)$ that will not be thrown to the atmosphere per year in this city.

We plan to extend the study to large scale instances, thus, we are interested in creating instances as close as possible to a whole city. This is a scientific challenge that could be faced using specially designed operators for this particular problem.

\section{ACKNOWLEDGEMENTS}

This research is partially funded by the Spanish Ministry of Economy and Competitiveness and FEDER under contract TIN2011-28194 and fellowship BES-2012-055967. It is also partially founded by project $8.06 / 5.47 .4142$ in collaboration with the VSB-Tech. Univ. of Ostrava and Universidad de Málaga, Andalucía Tech.

\section{REFERENCES}

[1] E. Alba, G. Luque, J. García-Nieto, G. Ordoez, and G. Leguizamón Mallba: a software library to design efficient optimisation algorithms. International Journal of Innovative Computing and Applications, 1(1):74-85, 2007.

[2] C. Blum and A. Roli. Metaheuristics in combinatorial optimization: Overview and conceptual comparison. ACM Computing Surveys (CSUR), 35(3):268-308, 2003.

[3] E. Brockfeld, R. Barlovic, A. Schadschneider, and M. Schreckenberg Optimizing traffic lights in a cellular automaton model for city traffic. Phys. Rev. E, 64(5):056132, Oct 2001

[4] M. Clerc and J. Kennedy. Standard PSO 2011. Technical Report http://www.particleswarm.info/, Particle Swarm Central, January 2011.

\footnotetext{
${ }^{2}$ Source: Sistema de Indicadores Urbanos Agenda 21. page 18. Observatorio de Medio Ambiente Urbano. Ayto. Malaga
}

[5] J. García-Nieto, E. Alba, and A. Carolina Olivera. Swarm intelligence for traffic light scheduling: Application to real urban areas. Engineering Applications of Artificial Intelligence, 25(2):274 - 283, 2012.

[6] Jose Garcia-Nieto, Ana Carolina Olivera, and Enrique Alba. Optimal Cycle Program of Traffic Lights With Particle Swarm Optimization. IEEE Trans.on Evolu. Comp., 17(6):823-839, December 2013.

[7] P. Holm, D. Tomich, J. Sloboden, and C. Lowrance. Traffic analysis toolbox volume iv: Guidelines for applying corsim microsimulation modeling software. Technical report, National Technical Information Service, 2007

[8] S. Kachroudi and N. Bhouri. A multimodal traffic responsive strategy using particle swarm optimization. In Symposium on Transportation Systems, pages 531-537, Redondo Beach, CA, USA, 2009.

[9] M. Keller. Handbook of Emission Factors for Road Transport (HBEFA) 3.1. quick reference. Technical report, INFRAS, 2010.

[10] J. Kennedy and R. C. Eberhart. Swarm Intelligence. Morgan Kaufmann Publishers, San Francisco, California, 2001.

[11] D. Krajzewicz, M. Bonert, and P. Wagner. The open source traffic simulation package SUMO. RoboCup 2006 Infrastructure Simulation Competition, 2006

[12] Stefan Lämmer and Dirk Helbing. Self-control of traffic lights and vehicle flows in urban road networks. Journal of Statistical Mechanics: Theory and Experiment, 1(04):4-19, April 2008.

[13] X. Li, G. Li, S. Pang, X. Yang, and J. Tian. Signal timing of intersections using integrated optimization of traffic quality, emissions and fuel consumption: a note. Transportation Research Part D: Transport and Environment, 9(5):401 - 407, 2004.

[14] J. McCrea and S. Moutari. A hybrid macroscopic-based model for traffic flow in road networks. European Journal of Operational Research, 207(2):676-684, 2010.

[15] J. Sánchez Medina, M. Galán Moreno, and E. Rubio Royo. Applying a Traffic Lights Evolutionary Optimization Technique to a Real Case: Las Ramblas Area in Santa Cruz de Tenerife. IEEE Trans. on Evo. Comp., 12(1):25 -40, 2008.

[16] Takashi Nagatani. Effect of speed fluctuations on a green-light path in a 2D traffic network controlled by signals. Physica A: Statistical Mechanics and its Applications, 389(19):4105-4115, October 2010.

[17] L. Peng, M. Wang, J. Du, and G. Luo. Isolation niches particle swarm optimization applied to traffic lights controlling. In 48th IEEE Conference on Decision and Control and 28th Chinese Control Conference, pages 3318 -3322, December 2009.

[18] K. Price, R.M. Storn, and J.A. Lampinen. Differential Evolution: A Practical Approach to Global Optimization. Natural Computing. Springer, 2005.

[19] N. M. Rouphail, B. B. Park, and J. Sacks. Direct signal timing optimization: Strategy development and results. Technical report, In XI Pan American Conference in Traffic and Transportation Engineering, 2000.

[20] D. J. Sheskin. Handbook of Parametric and Nonparametric Statistical Procedures. Chapman \& Hall/CRC, 2007.

[21] A. M. Turky, M. S. Ahmad, M. Z. Yusoff, and B. T. Hammad. Using genetic algorithm for traffic light control system with a pedestrian crossing. In RSKT '09: Proceedings of the 4th International Conference on Rough Sets and Knowledge Technology, pages 512-519, Berlin, Heidelberg, 2009. Springer-Verlag. 